Documentation et bibliothèques

DOCUMENTATION BIBLIOTHËQUES

\title{
Les services multiculturels de la Bibliothèque nationale du Canada
}

\section{André Houde}

Volume 27, numéro 1, mars 1981

URI : https://id.erudit.org/iderudit/1053830ar

DOI : https://doi.org/10.7202/1053830ar

Aller au sommaire du numéro

Éditeur(s)

Association pour l'avancement des sciences et des techniques de la documentation (ASTED)

ISSN

0315-2340 (imprimé)

2291-8949 (numérique)

Découvrir la revue

Citer cet article

Houde, A. (1981). Les services multiculturels de la Bibliothèque nationale du Canada. Documentation et bibliothèques, 27(1), 31-34.

https://doi.org/10.7202/1053830ar

Tous droits réservés (C) Association pour l'avancement des sciences et des techniques de la documentation (ASTED), 1981
Ce document est protégé par la loi sur le droit d'auteur. L'utilisation des services d'Érudit (y compris la reproduction) est assujettie à sa politique d'utilisation que vous pouvez consulter en ligne.

https://apropos.erudit.org/fr/usagers/politique-dutilisation/ 


\section{chronique}

\section{Les services multiculturels de la Bibliothèque nationale du Canada}

Rien de plus simple, semble-t-il, que la notion de services de bibliothèques, telle qu'exposée par les manuels. N'est-il pas à la fois facile et même amusant de répartir les diverses activités d'une bibliothèque dans les cases d'un organigramme bien conçu, la référence ici, le prêt entre bibliothèques là, le tout dans un enchaînement bien hiérarchisé ? Mais lorsqu'il faut marier services et clientèle, organigramme et réalité, les choses se compliquent: pour une bibliothèque universitaire, répondre aux besoins de programmes de niveaux distincts; pour une bibliothèque publique, rejoindre toutes les catégories sociales, des personnes d'âge, d'éducation et d'intérêts différents; pour une bibliothèque d'entreprise, satisfaire les économistes sans négliger les ingénieurs.

Si ce n'est pas une sinécure de servir une population hétérogène par ses besoins et ses intérêts, que dire des situations où la bibliothèque doit en plus le faire en deux ou plusieurs langues? Ces contextes bilingues ou multilingues sont fort nombreux au Canada, et plusieurs bibliothèques ne peuvent $y$ échapper. On oublie souvent, surtout au Québec où les traditions sont plus homogènes, que le Canada est une nation d'immigrants, et que leurs vagues successives se sont montrées de moins en moins assimilables, surtout depuis la période du dernier après-guerre, parce que constituées d'individus plus instruits, plus politisés, et donc plus enclins à vouloir sauvegarder leur culture d'origine.

Les bibliothèques canadiennes n'ont pas attendu les dernières décennies pour prendre conscience de ce phénomène et essayer d'y faire face. Déjà, en 1933, un rapport sur les bibliothèques canadiennes - le Rapport Ridington ${ }^{1}$ - soulignait la diversité d'origine des

1. Libraries in Canada; a Study of Library Conditions and Needs, by the Commission of Enquiry, John Ridington, Chairman, Toronto, Ryerson Press; Chicago, American Library Association, 1933 , p. $65,75,83$. populations que les bibliothèques de toutes les provinces, et en particulier du Manitoba, de la Saskatchewan et de l'Alberta, avaient à servir. On réalisait en même temps que la plupart des bibliothèques publiques n'avaient pas les ressources nécessaires pour répondre aux besoins de groupes variés et dispersés. Seules les plus importantes parmi les bibliothèques municipales pouvaient se permettre de mettre sur pied des services adéquats, comme la Toronto Public Library qui établissait en 1957 un Foreign Languages Center et fondait un peu plus tard une succursale entièrement chinoise ${ }^{2}$.

II fallut attendre encore quelque dix ans, jusqu'à la publication du Rapport de la Commission Laurendeau-Dunton, pour engendrer un processus de prise de conscience du problème aboutissant à des réalisations concrètes à l'échelle nationale. La Commission recommandait, dans le dernier volume de son rapport portant sur l'apport culturel des groupes ethniques canadiens (autres que le français et l'anglais)

que les organismes fédéraux, provinciaux et municipaux appropriés soient pourvus des moyens financiers nécessaires pour maintenir et augmenter leur aide aux associations culturelles et aux associations de recherche dont les objectifs consistent à développer les arts et les lettres des groupes culturels ni britanniques ni français ${ }^{3}$.

C'était reconnaître et affirmer que, vu la présence de deux langues officielles, le Canada n'a pas en tant que nation de culture unique.

La philosophie de ce rapport devait

2. Marie F. Zielinska, "Multiculturalism and library services to ethnic communities", Unesco Bulletin for Libraries, vol. 32 , no. 1 (Jan.-Feb. 1978), 18.

3. Commission royale d'enquête sur le bilinguisme et le biculturalisme, Rapport. Livre IV : l'apport culturel des autres groupes ethniques, Ottawa, 1969, p. 232 (recommandation no 14). 
influencer à la fois les milieux bibliothéconomiques et politiques et engendrer des initiatives séparées qui allaient éventuellement se rejoindre.

Le mérite des premières démarches pour établir un service national multilingue revient à la Canadian Library Association qui, dès 1969, procédait à un inventaire des ressources en langues étrangères des bibliothèques municipales canadiennes, révélant que $72 \%$ des bibliothèques municipales avaient besoin d'aide financière pour répondre aux besoins des différents groupes ethniques. Ce rapport ne devait pas rester lettre morte. Dès l'année suivante, en 1970, lors de son 25 e congrès annuel tenu à Hamilton, l'association adoptait une résolution invitant la Bibliothèque nationale du Canada à rendre accessibles aux 72 groupes ethniques canadiens - selon Statistique Canada - des collections dans leur langue à travers un réseau de bibliothèques publiques. La Bibliothèque nationale se mettait immédiatement à la tâche en établissant un comité ad hoc chargé d'étudier les mécanismes d'implantation d'un tel service. II s'agit du Rapport Wertheimer, remis le 27 mars 1971, lequel présentait le projet comme réalisable. Or, par une coïncidence qui illustrait bien l'importance croissante accordée par les Canadiens aux minorités de langues non officielles, le gouvernement fédéral faisait connaître presqu'en même temps, par la voix du premier ministre Pierre Elliot Trudeau - le 8 octobre 1971 - les grandes lignes de sa politique du multiculturalisme.

C'est finalement en 1973, après quelque 18 mois de préparation, d'études et de planification, que fut officiellement établi le Biblioservice multilingue de la Bibliothèque nationale du Canada ${ }^{4}$. Mais ce n'est qu'après avoir établi ses structures, recruté son personnel et créé un premier réseau de distribution lors d'une conférence nationale des bibliothécaires provinciaux à la Bibliothèque nationale du Canada en février $1974^{5}$ qu'il commençait vraiment ses opérations, le 19 mars $1975^{6}$.

On imagine facilement la complexité d'une telle organisation. Les difficultés se situaient à tous les niveaux de la mission confiée au service: choisir, cataloguer, traiter et distribuer des documents dans les langues des groupes ethniques canadiens.

Un service de ce genre ne se bâtit que peu à peu. II fallait d'abord décider quels groupes on desservirait en premier lieu, puisqu'il n'était

4. Bibliothèque nationale du Canada, Rapport annuel du directeur général de la Bibliothèque nationale 1975/76, Ottawa, 1976, p. 31.

5. Bibliothèque nationale du Canada, Rapport annuel $d u$ directeur général de la Bibliothèque nationale 1974/75, Ottawa, 1975, p. 12.

6. National Library of Canada, Launching of Multilingual Biblioservice; Remarks by the National Librarian, March 19, 1975, 4p. (Public Relations Office, News release). Aussi, de la même source: The National Librarian, Dr. Guy Sylvestre, launches the Multilingual Biblioservice, $3 \mathrm{p}$. évidemment pas possible de développer d'un coup des collections en 72 langues différentes. II était logique de commencer par les groupes numériquement les plus importants; les premières collections mises sur pied furent donc en chinois, hollandais, italien, polonais, espagnol, portugais, allemand et ukrainien. Mais comme le service s'adressait à tous les Canadiens, il fallait aussi aller au devant de groupes moins considérables, sous peine de ne répondre qu'à l'appel des groupes les mieux organisés. Aussi, dès 19767, le service distribuait-il des collections en 17 langues, ajoutant aux 8 langues du début l'arabe, le finlandais, le gaélique, le grec, l'irlandais, le hongrois, le maltais, le russe et le suédois. S'ajoutèrent aux cours des années subséquentes, toujours dans le but de servir le plus de groupes possible, le slovaque, le tchèque, le norvégien, l'hindu, l'urdu et le punjabi8.

II fallait ensuite déterminer les catégories et les types de documents à acquérir, et pour cela tenir compte non seulement de la composition démographique de chacun des groupes ethniques, mais encore de leurs niveaux d'éducation, de leurs habitudes culturelles, de leur degré d'assimilation, de leur intérêt pour la langue ancestrale. Le recensement de 1976 montrait par exemple que les Italiens venaient en tête des 8 premiers groupes ethniques selon le critère numérique, mais d'après les statistiques du service, ils ne venaient qu'au $5 e$ rang quant aux demandes d'emprunt, tandis que les Hollandais, qui n'occupaient que le $6 e$ rang par l'importance, venaient au premier rang par le nombre de demandes ${ }^{9}$. II s'avérait donc que les populations à desservir présentaient des caractéristiques et des intérêts différents dont il fallait tenir compte dans l'équilibre des collections.

On parvint, à la suite de ces études, à la formule générale suivante. Les collections seraient surtout de niveau populaire, composées à $40 \%$ de romans, $30 \%$ de littérature de jeunesse, et $30 \%$ de sujets divers, tels que biographies, livres de jardinage, livres de cuisine, etc.

La nature des collections une fois déterminée, il s'imposait d'établir les mécanismes de choix et d'acquisition. Encore ici, il est évident qu'un service d'une dizaine de personnes, même si elles sont toutes polyglottes - le personnel original maîtrisait au total 14 langues ${ }^{10}$ - ne pouvait couvrir la production mondiale dans une trentaine de langues. II fallut donc recourir à des contractuels. Les canaux de sélection utilisés

7. Marie Zielinska, “Multilingual Biblioservice; libraries in the Canadian mosaic", Canadian Library Journal, vol. 33, no. 5 (Oct. 1976), 443

8. "Canada: Multilingual Biblioservice of the National Library", Bookbird, vol. 14, no. 4 (1976), 40.

9. National Library of Canada, The Multilingual Biblioservice Enters its Sixth Year of Operation, Ottawa, 1979, p. 2.

10. "Multilingual Biblioservice starts to operate", National Library News, vol. 6, no. 3 (May-June 1974), 9. 
furent des plus divers: libraires spécialisés, bibliographies nationales, publications commerciales, systèmes d'envois d'office, dons des ambassades, choix fait parmi les documents envoyés d'un peu partout au Centre canadien d'échange du livre ${ }^{11 .}$

Les documents une fois choisis, il fallait les cataloguer et les classifier. Tant à cause de la complexité des opérations due au caractère multilingue des collections que par souci d'efficacité, on décida d'utiliser des procédures simplifiées : ainsi, pour le catalogage, on substitue aux vedettes-matière traditionnelles une annotation bilingue permettant au bibliothécaire ne connaissant pas la langue de l'ouvrage d'avoir au moins un aperçu de son contenu. De même, la classification se fait selon le système Dewey, plus simple et plus adapté aux bibliothèques publiques.

Enfin se posait le problème de la distribution. On rencontre ici, comme en bien des domaines au Canada, la question de la juridiction. Les bibliothèques étant de ressort provincial, la Bibliothèque nationale du Canada ne pouvait pas négocier directement avec les bibliothèques municipales. II fallut établir des ententes avec chacune des provinces afin que celles-ci se chargent, par l'intermédiaire d'organismes provinciaux, comme par exemple les bibliothèques centrales de prêt, d'établir un réseau de distribution. II y avait déjà, dès 1975, un réseau de 26 centres régionaux ${ }^{12}$. Le Biblioservice multilingue ne prête qu'à ces centres, et non directement aux bibliothèques municipales ou aux individus. Ces centres à leur tour se chargent de distribuer les collections, par rotation, aux diverses bibliothèques municipales qui en font la demande. La période de prêt peut varier de 6 mois à 3 ans. Le service est gratuit, en ce sens que la Bibliothèque nationale absorbe tous les frais de transport d'Ottawa aux centres régionaux. Ces derniers sont en revanche responsables des frais de transport à travers la province, ainsi que des réparations mineures indispensables. La Bibliothèque nationale fournit aussi aux centres un service gratuit de publicité (affiches, dépliants, etc.).

II faut remarquer que cette structure décentralisée présente de multiples avantages puisqu'elle permet aux bibliothèques municipales, qui ne peuvent se payer le luxe de spécialistes, de s'en remettre à la Bibliothèque nationale pour le choix et le traitement des collections, et que d'autre part elle laisse aux centres régionaux le soin de déterminer les localisations successives des collections, répondant ainsi davantage aux besoins, puisque ces centres peuvent mieux suivre la mobilité des groupes ethniques à

11. National Library of Canada, The Multilingual Biblioservice, Ottawa, n.d., p. 3.

12. Marie Zielinska, "Multilingual Biblioservice...", 443. l'intérieur d'une région ou d'une cité. Ainsi, un envoi de 2500 volumes au centre régional de Vancouver sera distribué par celui-ci à 7 succursales ${ }^{13}$.

Les collections du Biblioservice multilingue se limitent pour le moment aux monographies. La complexité de ses opérations est déjà assez grande sans y ajouter les problèmes causés par les périodiques. Cependant, le service a l'intention dans un avenir prochain d'élargir l'éventail des documents disponibles, en incluant dans ses collections l'audiovisuel, si important pour l'apprentissage des langues, et les documents adaptés aux handicapés, comme les livres en braille et les livres en gros caractères.

On voit par là que les problèmes posés par une telle entreprise ne sont pas à minimiser. $A$ eux seuls les problèmes techniques présentent des difficultés peu communes - pensons à l'acquisition de machines à écrire avec caractères chinois, ukrainiens, etc. 14 - et dans ce cas particulier même l'automatisation ne peut aider à résoudre tous les problèmes, puisque les ordinateurs ne peuvent pas encore lire les alphabets non romains.

Le Service multilingue a connu un essor fulgurant depuis sa fondation, preuve qu'il répondait à des besoins réels. II faisait 11000 prêts dès sa première année d'existence ${ }^{15}$, et ses collections totales passaient entre 1975 et 1978 de 25000 à 90000 volumes ${ }^{16}$, atteignant en 19791980 un total de 100000 volumes en 26 langues, dont l'estonien, le lithuanien et le gallois. Fait intéressant à noter, plus d'un tiers des prêts vont à l'Ontario, suivie de la Colombie britannique, du Québec, du Manitoba et de la Saskatchewan ${ }^{17}$.

Le service n'a pas voulu se limiter aux prêts de collections. II s'est aussi donné une vocation bibliographique en profitant, là où elle s'applique, des bienfaits de la loi du dépôt légal. II vise à compiler une liste de tous les périodiques canadiens dans les langues autres que le français et l'anglais, qu'il s'agisse de journaux ou de revues. C'est ainsi qu'en 1976, il établissait une liste, divisée entre 41 groupes culturels, de 170 titres courants de journaux ethniques reçus par la Bibliothèque nationale, et une autre liste, alphabétique, de 350 titres courants et rétrospectifs (on sait que le premier journal ethnique canadien est un journal de langue

13. "Cover photo", BCLA Reporter, vol. 19, no. 5 (Jan.-Feb. 1976), 2.

14. Ruth Gordon, "Members of minority groups", Canadian Scene, June 27, 1975.

15. F. Dolores Donnelly, "Canada's national library services", in Canadian Libraries in their Changing Environment, ed. by L.S. and C. Garry, Toronto, York University, Center for Continuing Education, 1977, p. 289.

16. Bibliothèque nationale du Canada, Rapport annuel du directeur général de la Bibliothèque nationale 1978/79, Ottawa, 1979, p. 49.

17. National Library of Canada. The Multilingual Biblioservice Enters..., p. 2. 
allemande publié en Ontario en 1835). Les journaux ethniques canadiens sont d'une importance capitale pour la connaissance de ces groupes, se rapprochant plutôt du périodique sans pourtant en adopter la présentation ${ }^{18}$. Le service veut aussi établir la liste des revues ethniques canadiennes. Une première liste d'environ 600 titres a déjà été dressée - ce sont les titres faisant partie des collections de la Bibliothèque nationale. Cependant, on pense que quelque 2000 titres de revues ethniques auraient été publiées au Canada depuis 1788 . II y a donc un énorme travail de récupération à accomplir. D'autre part, le service voit aussi au microfilmage de ces documents pour en assurer la préservation, souvent dans le cadre de programmes coopératifs, comme avec l'University of British Columbia pour les journaux chinois ou I'Ontario Council of University Libraries pour les journaux ontariens. Enfin, le service pense établir un catalogue collectif de ces publications, afin d'en connaître exactement toutes les localisations canadiennes ${ }^{19}$.

Comme le faisait remarquer avec humour Madame Marie Zielinska, directrice du service, le Biblioservice multilingue va théoriquement à l'encontre des buts poursuivis par la Bibliothèque nationale. Alors que celle-ci a pour mission essentielle de recueillir les documents canadiens, le service a pour but de disséminer des documents surtout étrangers 20 . Ce paradoxe n'est évidemment qu'apparent, puisque la fonction fondamentale de la Bibliothèque nationale est de répondre aux besoins des Canadiens en s'adaptant à leur réalité sociologique. Or ceux-ci sont encore une nation d'immigrants. La Bibliothèque nationale doit ainsi s'inscrire dans le courant de réveil des valeurs ethniques tendant à mettre en valeur les différences culturelles malgré les progrès assimilateurs de l'urbanisation, de la technologie et des communications de masse ${ }^{21}$, de telle sorte que les enfants des immigrants puissent à la première génération s'adapter à leur milieu et à la $2 e$ et $3 e$ réussir à préserver leur héritage propre. L'expérience du service est d'autant plus louable qu'il constitue le premier organisme du genre au monde qui soit promu par un gouvernement, et qu'il se situe dans un ensemble de services pionniers, comme l'Office national du film, les Musées nationaux, les Archives publiques, autant d'institutions canadiennes qui essaient de promouvoir les valeurs ethniques ${ }^{22}$. II a suscité des imitateurs en Grande-Bretagne, en Australie, au Danemark, en Suède, en Allemagne et aux Pays-Bas ${ }^{23}$, et il ne

18. "Le nouveau programme multiculturel de la Bibliothèque nationale", Nouvelles de la Bibliothèque nationale, vol. 4, no 1 (janv.-fév. 1972), 8.

19. Marie Zielinska, "Multilingual Biblioservice...", 441.

20. Ibid., 442 .

21. Marie F. Zielinska, "Multiculturalism and library services...", 17.

22. Ibid., 18

23. Ibid., 20-21. fait pas de doute que la Bibliothèque nationale du Canada a l'intention dans l'avenir non seulement de le soutenir mais aussi de le développer ${ }^{24}$.

II ne reste, en terminant, qu'à souhaiter que l'une des recommandations du rapport Wertheimer soit appliquée par toutes les écoles de bibliothéconomie du Canada:

que les écoles de bibliothéconomie soient informées de la valeur et de l'importance des services en langues étrangères des bibliothèques canadiennes, afin qu'elles puissent établir un programme de formation qui tienne compte de cette réalité 25 .

\author{
André Houde \\ Programme de bibliotechnique \\ Collège Algonquin \\ Ottawa
} 24. Bibliothèque nationale du Canada, L'avenir de la Bibliothè-
que nationale du Canada, Ottawa, 1979, p. 23.

25. "Le nouveau programme multiculturel...", 6-7. 